\title{
Reflection contributions to the dispersion artefact in FTIR spectra of single biological cells $\dagger$
}

\author{
Paul Bassan, ${ }^{a}$ Hugh J. Byrne, ${ }^{* b}$ Joe Lee, ${ }^{a}$ Franck Bonnier, ${ }^{b}$ Colin Clarke, ${ }^{b}$ Paul Dumas, ${ }^{c}$ Ehsan Gazi, ${ }^{d}$ \\ Michael D. Brown, ${ }^{d}$ Noel W. Clarke ${ }^{d e f}$ and Peter Gardner ${ }^{a}$
}

\author{
Received 1st December 2008, Accepted 2nd April 2009 \\ First published as an Advance Article on the web 9th April 2009 \\ DOI: $10.1039 / b 821349 f$
}

Fourier transform infrared spectra of a single cell in transflection geometry are seen to vary significantly with position on the cell, showing a distorted derivative-like lineshape in the region of the optically dense nucleus. A similar behaviour is observable in a model system of the protein albumin doped in a potassium bromide disk. It is demonstrated that the spectrum at any point is a weighted sum of the sample reflection and transmission and that the dominance of the reflection spectrum in optically dense regions can account for some of the spectral distortions previously attributed to dispersion artefacts. Rather than being an artefact, the reflection contribution is ever present in transflection spectra and it is further demonstrated that the reflection characteristics can be used for cellular mapping.

\section{Introduction}

Fourier transform infrared (FTIR) spectroscopy has been demonstrated as a potential tool for disease diagnostics in tissue $^{1-3}$ and in its microscopical form, for cellular analysis. ${ }^{\text {4-12 }}$ The use of synchrotron sources and attenuated total reflection imaging have achieved subcellular resolution thus promising a powerful technique for the analysis of the biochemical origin of disease. ${ }^{7,13,14}$ However, both tissue and cellular material are extremely spatially inhomogeneous on a micrometre scale in terms of physical morphology and chemical content and such inhomogeneity can give rise to elastic scattering of the incident radiation which is the origin of a broad undulating background to spectra which have been attributed to Mie scattering. ${ }^{15}$ In the case of cells, the cytoplasm is relatively sparse whereas the nucleus is rather dense in terms of biochemical content giving rise to a misrepresentation of the relative densities of biochemical constituents. ${ }^{16,17}$ These effects can lead to many ambiguous results depending on the geometry of the measurement. In diffuse reflection infrared Fourier transform (DRIFT) or transflection

${ }^{a}$ School of Chemical Engineering and Analytical Science, Manchester Interdisciplinary Biocentre, The University of Manchester, 131 Princess Street, Manchester, UK M17DN.E-mail:Peter.Gardner@Manchester. ac.uk

${ }^{b}$ Focas Research Institute, Dublin Institute of Technology, Kevin Street, Dublin 8, Ireland

'Synchrotron SOLEIL, L'Orme des Merisiers, BP48 - Saint Aubin, 91192 Gif-sur-Yvette Cedex, France

${ }^{d}$ Genito Urinary Cancer Research Group, School of Cancer and Imaging Sciences Paterson Institute for Cancer Research, University of Manchester, Wilmslow Road, Manchester, UK M20 4BX

${ }^{e}$ Department of Urology, The Christie NHS Foundation Trust, Manchester, UK M20 4BX

${ }^{f}$ Department of Urology, Salford Royal NHS Foundation Trust, Salford, UK M6 $8 H D$

$\uparrow$ This paper is part of an Analyst themed issue on Optical Diagnosis. The issue includes work which was presented at SPEC 2008 Shedding Light on Disease: Optical Diagnosis for the New Millennium, which was held in São José dos Campos, São Paulo, Brazil, October 25-29, 2008. modes 'contamination by contributions of reflection' can lead to further spectral distortions or so-called 'dispersion artefacts'. ${ }^{18}$

These confounding factors can result in significant frequency shifting of spectral features used to classify biological species for diagnosis or biochemical analysis. It has been demonstrated that the broad Mie scattering contribution can be modelled ${ }^{\mathbf{1 6}}$ and even removed in an automated fashion using the Extended Multiplicative Signal Correction (EMSC) algorithm. ${ }^{19}$ The reflection 'contamination' or dispersion artefact can be corrected for by back-transforming the spectra and extracting the real and imaginary components of the spectra resulting in a significantly reduced dispersive components. ${ }^{15}$

In order to progress the potential of FTIR spectroscopy for biomedical applications it is imperative that the true origin of these factors be understood. This is especially true in an interdisciplinary field spanning the physical and life sciences and even the clinical community where terms such as 'contamination' and 'artefact' mask the true origin of the observed effects.

In this paper it is demonstrated that the spectral distortions observable in FTIR spectra in spatially inhomogeneous samples in a transflection geometry can have origin in a variation of the relative contributions of the reflection and transmission spectra of the material and that at high optical densities the spectrum can be dominated by the reflection component. In cellular spectra, far from being an artefact or contaminant, the pure reflection spectrum provides a biochemical map of the surface, free from artefacts, yielding subcellular information on the nucleus and cytoplasm.

\section{Experimental}

Human metastatic prostate cancer (PC-3) cells were cultured onto calcium fluoride $\left(\mathrm{CaF}_{2}\right)$ windows and fixed according to a protocol outlined by Gazi et al. ${ }^{9}$ A reflection line map was measured at the SOLEIL synchrotron facility (SMIS beamline) ${ }^{\mathbf{2 0}}$ to take advantage of the high brilliance of the source for small spot sizes. The spectra were recorded using the SMIS beamline and end-station which has been described elsewhere. ${ }^{20}$ 
A projected aperture size of $10 \mu \mathrm{m} \times 10 \mu \mathrm{m}$ was used with a line map step size of $10 \mu \mathrm{m} .2048$ scans were co-added using a spectral resolution of $8 \mathrm{~cm}^{-1}$ to increase the signal-to-noise ratio as the reflection signal is inherently weak in magnitude.

As a model sample, a $6 \%$ dispersion of the protein albumin in a $\mathrm{KBr}$ matrix was produced. A $12 \mathrm{mg}$ sample of albumin was mixed with $188 \mathrm{mg}$ of $\mathrm{KBr}$ powder. The two powders were dried in a vacuum oven overnight to decrease as much as possible the contribution of water in the spectra before being pressed into a pellet.

A pellet containing only $\mathrm{KBr}(200 \mathrm{mg}$ ) was prepared and used as the reference sample for transmission measurements. A gold substrate was employed as a reference of $100 \%$ in reflection mode and transflection mode. While the pellets were freestanding for reflection and transmission measurements, in the case of transflection measurements the pellets were placed on a gold surface to obtain a full reflection of the infrared beam.

In the case of measurements of the model albumin $/ \mathrm{KBr}$ material, a Perkin Elmer Spotlight 400 was employed. The single point mode was used to record the spectra with an aperture size set at $100 \mu \mathrm{m} \times 100 \mu \mathrm{m}$. A map was defined with a step size of $100 \mu \mathrm{m} .32$ scans were co-added using a spectral resolution of $4 \mathrm{~cm}^{-1}$.

\section{Results and discussion}

As an example of the complexity of FTIR spectra which can be obtained from FTIR micro-spectroscopy in the transflection geometry, and the variability which can occur as a result of spatial inhomogeneities, Fig. 1 shows a spectral line map of a PC3 cell taken at $10 \mu \mathrm{m}$ step intervals. Measurements were taken in transflection mode and are expressed as absorbance. The background was recorded from a bare $\mathrm{CaF}_{2}$ disk and thus the spectrum indicates the changes in cellular reflection (specular and/or diffuse) relative to the bare $\mathrm{CaF}_{2}$. For all positions, the spectra are relatively noisy as the reflection from the cell/substrate interface is expected to be weak as the refractive index of the $\mathrm{CaF}_{2}$ substrate $\left(1.387 \text { at } 1696 \mathrm{~cm}^{-1}\right)^{21}$ is only slightly larger than that of the cellular material normally considered to be about 1.3. ${ }^{22}$ At position (b), the spot is large enough that it samples some of the cytoplasm and the transflection spectrum shows typical cellular features associated with proteins (Amide I $\sim 1650$ $\mathrm{cm}^{-1}$, Amide II $1565 \mathrm{~cm}^{-1}$ ). In the region of the nucleus, however, $(\mathrm{c}, \mathrm{d})$, the spectra have become significantly distorted and resemble a first-order derivative lineshape. It is these types of distortions which have previously been attributed to dispersion artefacts, and interpretation of the spectra for diagnostic applications or biochemical analysis is difficult as changes to physical morphology can mask changes in chemical composition.

The inhomogeneity of the cellular sample can be mimicked by a dispersion of the protein albumin loaded in a $\mathrm{KBr}$ matrix. Fig. 2a shows a microscopic image of the sample with $6 \%$ loading of albumin in $\mathrm{KBr}$. Within the matrix, the protein is inhomogeneously dispersed, mimicking the spatial inhomogeneity of a cellular material. Fig. $2 b$ maps the transflection spectrum, expressed as absorbance, as the measurement position is moved from a region of low albumin density to one of high density. Similar to the case of the cellular line map of Fig. 1, the spectrum becomes highly distorted in the regions of high density,

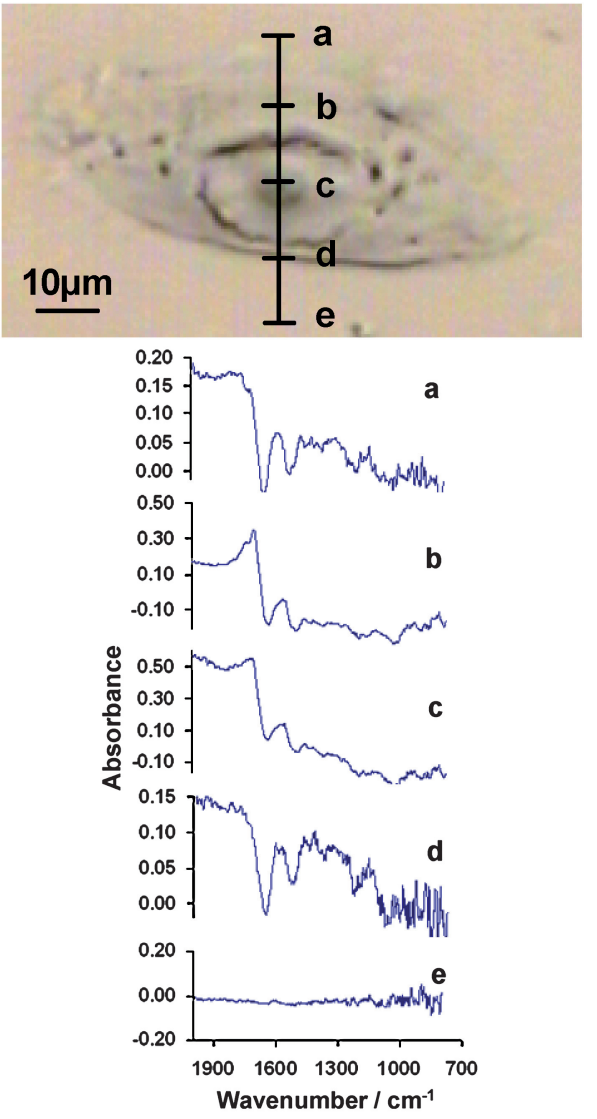

Fig. 1 (a) Optical image of PC-3 cell on a $\mathrm{CaF}_{2}$ substrate. (b) Spectra of the cell at the points indicated in (a) expressed as absorbance.

exhibiting distorted lineshapes in the region of the Amide I band characteristic of 'dispersion artefacts'. The positions of the absorption maxima at, for example $\sim 1675 \mathrm{~cm}^{-1}$ and $1560 \mathrm{~cm}^{-1}$, indicated by the vertical lines in Fig. 2b, clearly shift in frequency. In contrast to the case of the cellular spectra, however, the inhomogeneity is purely morphological and there can be no question of spectral changes due to variations in chemical composition.

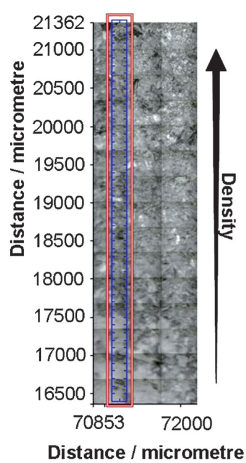

(a)

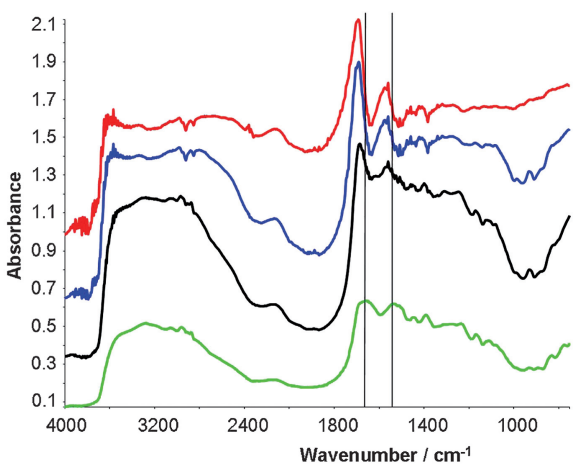

(b)
Fig. 2 (a) Microscope image of $6 \%$ loading albumin $/ \mathrm{KBr}$ pellet. The red/ blue rectangles indicate the regions spectroscopically sampled. (b) Variation in the FTIR transflectance spectrum, expressed as an absorbance as the sampled area varies from the low density to the high density region. 
The variations in the spectral form can be simply understood by considering the relative contributions to the spectrum of the transmitted and surface-reflected light. Transflection experiments are commonly performed by placing a sample on a highly reflective substrate, focusing an IR beam onto a region of interest and collecting the photons that are reflected back into the collection optics. The method is called transflection, because the majority of the signal collected is a transmission signal as the beam passes through the sample, reflects off the substrate, passing through the sample again to the detector as shown in Fig. 3. As the base substrate is highly reflecting across the spectral region of interest, it is assumed that the spectrum gathered is dominated by the transmission characteristics of the sample under investigation. However, the gathered signal also contains a contribution from the top interface between the air and the sample. The total signal can therefore be approximated by

$$
I_{\mathrm{Tf}}=2 T+R
$$

where $I_{\mathrm{Tf}}$ is the gathered transflection signal, $T$ the single pass transmission and $R$ the reflection. The transflection signal therefore consists of a weighted sum of the transmission and reflection characteristics of the sample. In samples where the optical density is low, the weighting is such that the transmission signal dominates and the reflection signal is negligible, producing spectra that closely represent pure transmission data. If the transmission signal is weak or extinct, however, the reflection signal will dominate and a reflectance spectrum is measured.

The optical properties of a material are governed by the complex refractive index $\eta$ (see, for example, ref. 19)

$$
\eta=n+i \kappa=n+i \alpha / 2 k_{0}
$$

where $\alpha$ is the absorption coefficient and $k_{0}$ is $2 \pi / \lambda_{0}$, such that as a function of driving frequency $\omega$ for a simple driven electric dipole oscillator of resonance frequency $\omega_{0}$,

$$
\begin{gathered}
n=1+\frac{N e^{2}}{4 \varepsilon_{0} m \omega_{0}} \frac{\left(\omega_{0}-\omega\right)}{\left(\omega_{0}-\omega\right)^{2}+(\gamma / 2)^{2}} \\
\alpha=\frac{N e^{2} \pi}{4 \varepsilon_{0} m c} \frac{\gamma / 2}{\left(\omega_{0}-\omega\right)^{2}+(\gamma / 2)^{2}}
\end{gathered}
$$

where $\gamma$ is the usual Lorentz damping factor.

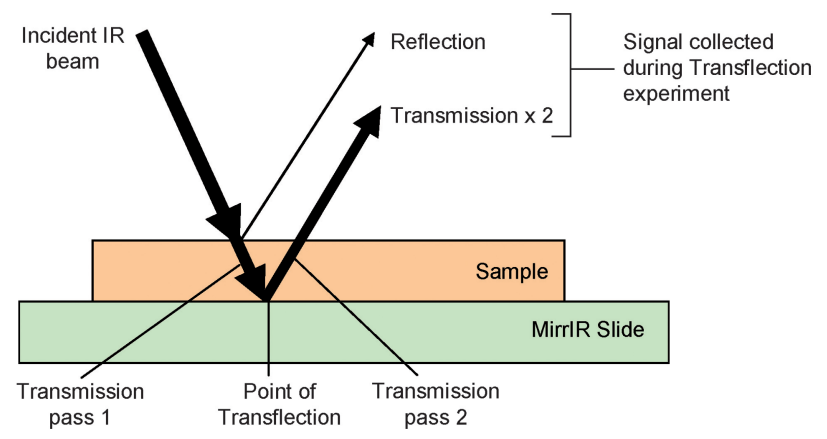

Fig. 3 Schematic of a typical transflection experiment. The input beam is drawn at an arbitrary incident angle for clarity.
Eqns (3) and (4) constitute the Kramers-Kronig dispersion relations between the absorption and dispersion and can be shown graphically as in Fig. 4. Eqn (3) is calculated for the case of gaseous media at low pressures such that the off resonant real component approximates to 1 . For the sake of the simulation of organic materials, this off resonant value is taken as 1.3 in this simulation.

The reflection from the interface of two materials, in the absence of absorption, is given by

$$
R=\left(\frac{n_{1}-n_{2}}{n_{1}+n_{2}}\right)^{2}
$$

where $n_{1}$ is the refractive index of the interface medium (in the case of interest here, air) and $n_{2}$ is that of the reflecting medium (cell). Assuming that $n_{2}$ has a value of 1.3, the non-resonant reflection of an air/cell interface can be calculated to be 0.017 or $1.7 \%$.

The sample transmission is given by

$$
T=\exp (-\alpha l)
$$

where $l$ is the thickness of the sample. Both the transmission and reflection signals are frequency dependent and the spectra are a manifestation of the electronic states within the material.

The effect of various weightings of the transmission and reflection signals can be simulated from the optical parameters of the idealized simple driven electric dipole oscillator shown in Fig. 4. Fig. 5 shows the variation of such a simulated transflection spectrum, expressed as absorbance (as would commonly be the case in an FTIR transflection measurement) as the relative weightings $R \%: T \%$ of the calculated reflection and transmissions are varied from $0 \%: 100 \%$ (bottom) to $100 \%: 0 \%$ (top). It is clear that by effectively changing the optical thickness of the 'sample' the spectral lineshape varies considerably, from being initially dominated by the absorption characteristics to being dominated by the reflection. In terms of the distortion of the pure absorbance feature, the increased relative contribution of the reflection component affects spectral changes similar to those

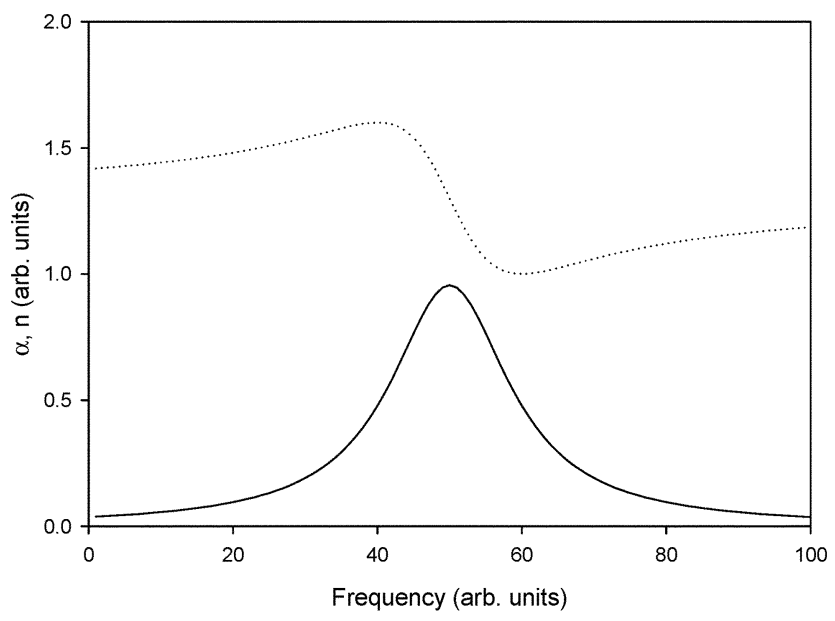

Fig. 4 Simulated absorption $\alpha(\omega)$ and dispersion $n(\omega)$, as a function of frequency $\omega$, in the region of a spectroscopic transition of resonance frequency $\omega_{0}=50$ (arb. units). 


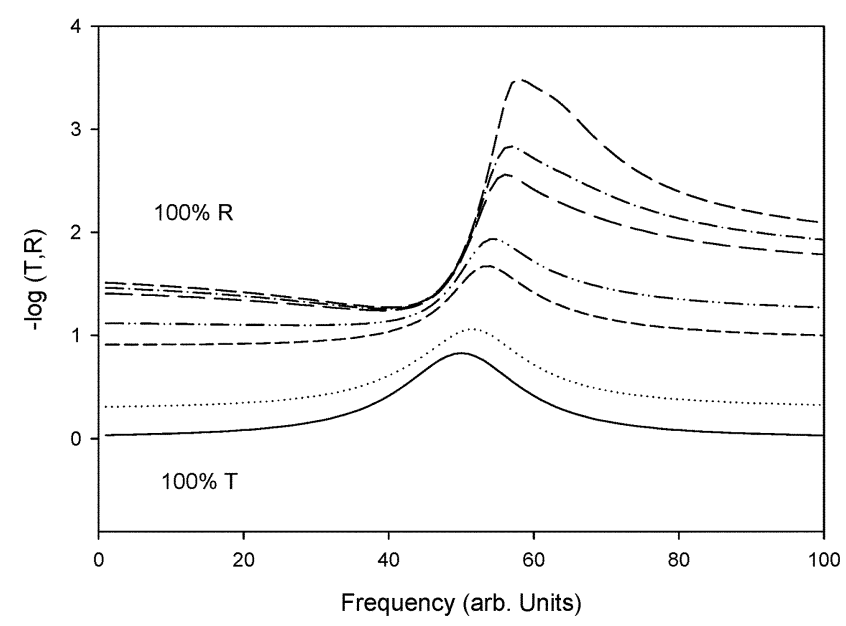

Fig. 5 Simulated variation in the spectral profile as a result of the weighted sum of the reflection and transmission from a simple harmonic oscillator with resonance frequency $\omega_{0}=50$ (arb. units).

termed the dispersion artefact. It is stressed, however, that this is not an artefact, but a contribution of the reflection due to the real component of the refractive index.

In the transflection geometry, therefore, the measured signal is a sum of the sample transmission and the reflection from the top surface. Fig. 6 compares the spectrum measured in transmission mode, expressed as absorbance, of the albumin/ $\mathrm{KBr}$ sample in regions of low density, with the transflection spectrum of the low density region of Fig. 2. An almost identical spectral match is found. In the high density regions, however, the spectrum becomes dominated by the reflection spectrum, as seen in Fig. 7. It should be noted that in such a spatially inhomogeneous system, diffuse reflectance may contribute as much as specular reflectance. The transflection spectrum matches almost identically with the spectrum measured in reflection mode. Although the quality of the spectra in terms of signal-to-noise varies considerably, the spectral shape in reflection mode is found to be independent of albumin density.

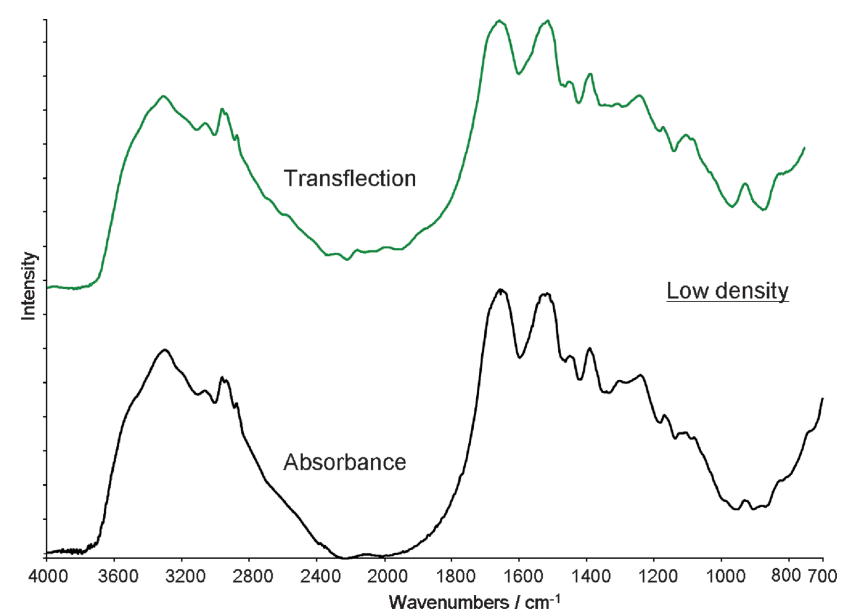

Fig. 6 Transflection of albumin/KBr sample as measured in the low density regions of the sample, compared with the absorbance as measured in transmission mode. The spectra are offset for clarity.

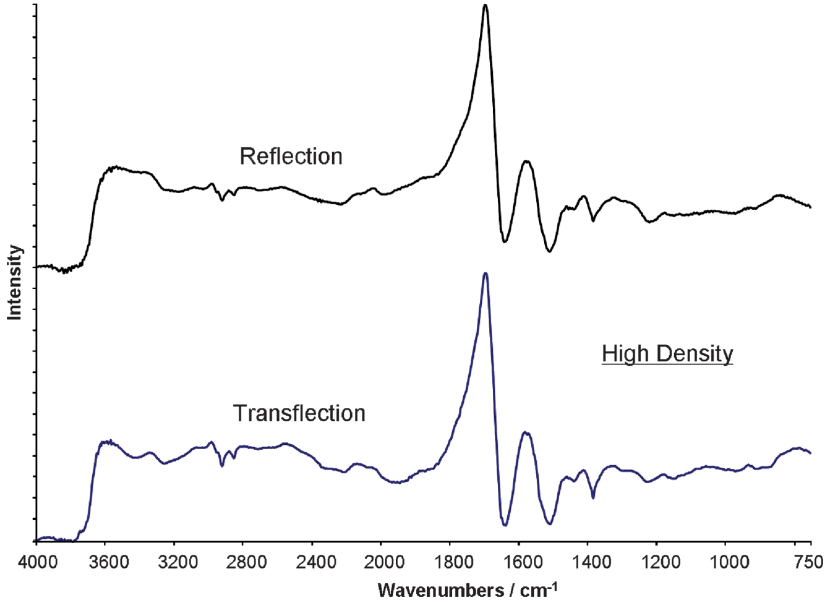

Fig. 7 Reflection spectrum of albumin/KBr plate compared with the transflection spectrum in the high density regions. Spectra are offset for clarity.

As shown in Fig. 8, in the intermediate regime, the transflection spectrum can be well fitted with a weighted sum of the high density reflection spectrum and the low density reflection spectrum such that

$$
I_{\mathrm{Tf}}=a T+b R
$$

In the examples presented in Fig. 1, a low reflectivity substrate, $\mathrm{CaF}_{2}$, was employed to minimise the transmission contribution to the transflection spectrum in order to highlight the contribution of the reflection spectrum. Normally, a high reflection ('low e') substrate such as MirrIR is employed to maximise the transmission component hence minimising the contribution of the reflection spectrum. However, this minimisation leads to the ever-present contribution being over-looked or dismissed as a 'contamination'. The reflection spectrum has origin in the chemical constituents of the material, however, and is potentially as valuable a characterisation tool as the material absorption.

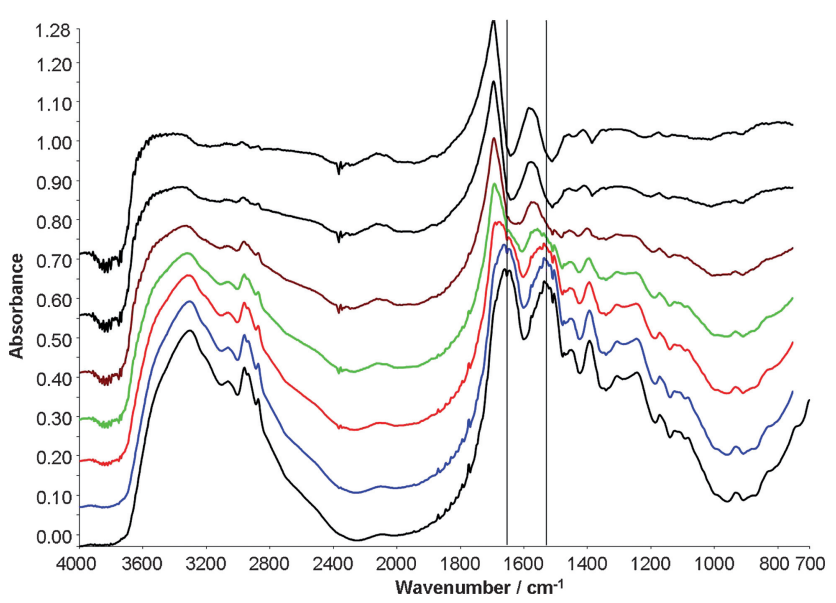

Fig. 8 Simulation of spatial variation of the transflection spectrum of albumin in $\mathrm{KBr}$ using the weighted sums of the reflection and transmission spectra, from $100 \% \mathrm{R}$ (top) to $100 \% \mathrm{~T}$ (bottom). 
Fig. 9 maps the $1240 \mathrm{~cm}^{-1}, \mathrm{PO}_{2}$, Phosphate DNA backbone, and protein $\mathrm{PO}_{2}$ groups, which should be dominant in the nucleus of the cell. Although the spectra in the region of the nucleus are dominated by the surface reflection, as indicated by the first derivative type lineshape, it is clear that the map contains information on the nucleus. In a dielectric material, the sampling depth of the pure reflection component is of the order of the wavelength of the incident radiation which in the region of the DNA bands at $1240 \mathrm{~cm}^{-1}$ corresponds to $\sim 8 \mu \mathrm{m}$. Such a penetration depth corresponds well with typical nuclear dimensions Rather than being a contamination, therefore, the suggestion is that the reflection spectrum of the material is a relatively uncontaminated representation of the biochemical content and variation of the sample under investigation, although it is acknowledged that the signal-to-noise is considerably compromised and therefore sampling times required would be significantly increased.

\section{Conclusions}

It has been demonstrated that, in a transflection geometry, a Fourier transform infrared spectrum is a weighted sum of the sample transmission and reflection spectra of the sample. The contribution of the reflection spectrum can become dominant in regions of high optical density and produces spectral distortions characteristic of the so-called 'dispersion artefact'. In the transflection geometry, the effect is in reality not dominated by dispersion and is not an artefact, and it has been shown that the reflection spectrum can be used as a biochemical mapping technique in its own right. Recognising that it is omnipresent to varying degrees in transflection spectra prompts a move to either reflection- or transmission-only geometries.

(a)

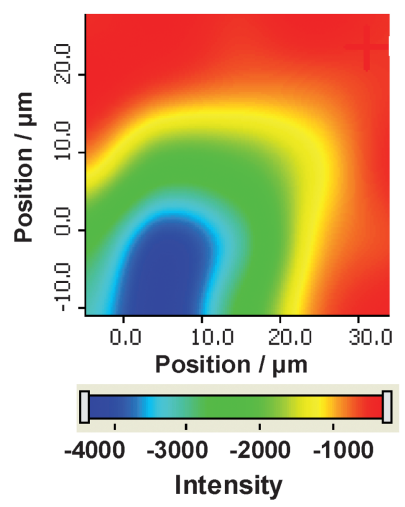

(b)

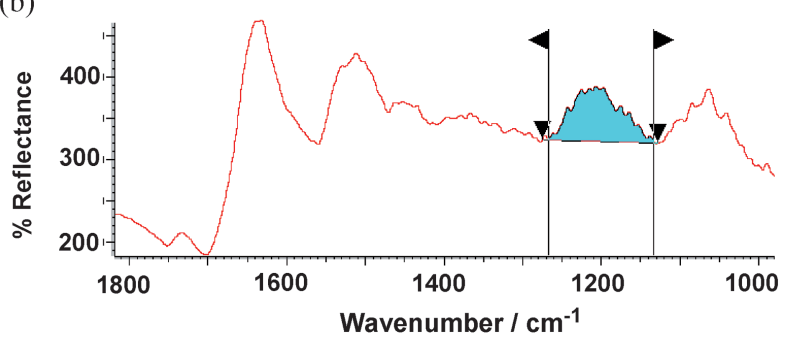

Fig. 9 (a) Map of a PC-3 cell on $\mathrm{CaF}_{2}$ based on the integrated band at $1240 \mathrm{~cm}^{-1}$ shown in (b). Note that blue indicates highest reflectance, red the lowest.
This study has taken an extreme example of a low reflectivity substrate to minimise the transmission contributions for illustrative purposes. In minimising the transmission contributions, transmission dispersive artefacts are also minimised. The origin of the dispersion artefact in purely transmission geometries is currently being explored. ${ }^{23}$ Such an elucidation could lead to an evolution of data processing protocols such as EMSC to fully incorporate resonant reflection and scattering contributions such that the real and imaginary components can be deconvolved, yielding a reliable and interpretable absorption spectrum of cellular samples.

\section{Acknowledgements}

This study derived from discussions of the Single Cell Spectroscopy Working Group of the EU FP6 SSA DASIM, Contract Number 005326. The work at DIT is associated with NBIPI, funded by the Irish Government's Programme for Research in Third Level Institutions, Cycle 4, National Development Plan 2007-2013.

\section{References}

1 R. Dukor, Vibrational spectroscopy in the detection of cancer, in Handbook of Vibrational Spectroscopy, ed. J. M. Chalmers and P. R. Griffiths, vol. 5, Wiley, Chichester, 2002, pp. 3335-3361.

2 E. Gazi, M. Baker, J. Dwyer, N. P. Lockyer, P. Gardner, J. H. Shanks, R. S. Reeve, C. Hart, N. W. Clarke and M. Brown, European Urology, 2006, 50, 750-761.

3 D. C. Fernandez, R. Bhargava, S. M. Hewitt and I. W. Levin, Nature Biotechnol., 2005, 23, 469-474.

4 N. Jamin, P. Dumas, J. Moncuit, W. H. Fridman, J. L. Teillaud, L. G. Carr and G. P. Williams, Proc. Natl. Acad. Sci. USA, 1998, 95, 4837-4840.

5 P. Lasch, A. Pacifico and M. Diem, Biopolymers, 2002, 67, 335-338.

6 P. Lasch, M. Boese, A. Pacifico and M. Diem, Vib. Specrosc., 2002, 28, 147-157.

7 P. Dumas and L. Miller, Vib. Spectrosc., 2003, 32, 3-21.

8 P. Dumas, N. Jamin, J. L. Teillaud, L. M. Miller and B. Beccard, Faraday Discuss., 2004, 126, 289-302.

9 E. Gazi, J. Dwyer, N. P. Lockyer, P. Gardner, J. Miyan, C. A. Hart, M. D. Brown, J. H. Shanks and N. W. Clarke, Biopolymers, 2005, 77, $18-30$.

10 E. Gazi, J. Dwyer, N. P. Lockyer, J. Miyan, P. Gardner, C. A. Hart, M. D. Brown and N. W. Clarke, Vib. Spectrosc., 2005, 38, 193-201.

11 E. Gazi, P. Gardner, N. P. Lockyer, C. A Hart, N. W. Clarke and M. D. Brown, J. Lipid Research, 2007, 48, 1846.

12 D. A. Moss, M. Keese and R. Pepperkok, Vib. Spectrosc., 2005, 38, 185-191.

13 P. Dumas and M. J. Tobin, Spectroscopy Europe, 2003, 15, 17-23.

14 P. Dumas, G. D. Sockalingum and J. Sulé-Suso, Trends in Biotechnology, 2007, 25, 40-44.

15 M. Romeo and M. Diem, Vib. Spectrosc., 2005, 38, 129-132.

16 M. Romeo, B. Mohlenhoff and M. Diem, Vib. Spectrosc., 2006, 42, $19-14$.

17 J. Lee, E. Gazi, J. Dwyer, M. D. Brown, N. W. Clarke, J. Nicholson and P. Gardner, Analyst, 2007, 132, 750-755.

18 M. Romeo and M. Diem, Vib. Spectrosc., 2005, 38, 115-119.

19 S. N. Thennadil, H. Martens and A. Kohler, Applied Spectroscopy, 2006, 60, 315-321.

20 P. Dumas, F. Polack, B. Lagarde, O. Chubar, J. L. Giorgetta and S. Lefrancois, Infrared Physics \& Technology, 2006, 49, 152-160.

21 CRC Handbook of Chemistry and Physics, ed. D. R. Lide, CRC Press Inc, Boca Raton, FL, 62nd edn, 1981.

22 A. Kohler, J. Sulé-Suso, G. D. Sockalingum, M. Tobin, F. Bahrami, Y. Yang, J. Pijanka, P. Dumas, M. Cotte, D. G. van Pittius, G. Parkes and H. Martens, Applied Spectroscopy, 2008, 62, 259-266.

23 P. Bassan, H. J. Byrne, F. Bonnier, J. Lee, P. Dumas and P. Gardner, Analyst, 2009, submitted. 